doi: $10.15407 /$ ujpe61.08.0690

G.P. BEREZINA, K.V. GALAYDYCH, R.R. KNIAZIEV, A.F. LINNIK, P.I. MARKOV, O.L. OMELAENKO, I.N. ONISHCHENKO, V.I. PRISTUPA, G.V. SOTNIKOV, A.P. TOLSTOLUZHSKY, V.S. US

National Science Center "Kharkiv Institute of Physics and Technology",

Nat. Acad. of Sci. of Ukraine

(1, Akademichna Str., Kharkiv 61108, Ukraine; e-mail: onish@kipt.kharkov.ua)

\title{
MULTIBUNCH REGIME OF WAKEFIELD PACS 52.35.Qz; 52.40.Kh EXCITATION IN A PLASMA-DIELECTRIC STRUCTURE
}

\section{Introduction}

While tackling the general problems of high-energy physics, electron accelerators play an important role of an instrument, like a microscope, for studying the tiniest world of the nature, whose dimensions can be observed to a trillionth of a micron making use of modern colliders with an energy of $100 \mathrm{GeV}$ in the center-of-mass system [1]. Today, we move forth into a new TeV-energy range $[2,3]$, where we expect to obtain answers to profound fundamental issues concerning the mass origin, prevalence of the matter over the antimatter, existence of supersymmetry, and others. Accelerators of high-energy ions, including proton and heavy-ion colliders [4], can reveal the in situ synthesis of a nuclear matter by means of the quarkgluon plasma creation at a temperature of the quarkhadron phase transition equal to about one trillion Kelvins, which is assumed to be the high-density state of the Universe at a moment of $10^{-5} \mathrm{~s}$ after the Big Bang.

(c) G.P. BEREZINA, K.V. GALAYDYCH, R.R. KNIAZIEV, A.F. LINNIK, P.I. MARKOV, O.L. OMELAENKO, I.N. ONISHCHENKO, V.I. PRISTUPA, G.V. SOTNIKOV, A.P. TOLSTOLUZHSKY, V.S. US, 2016
However, accelerators like the International Linear (Lepton) Collider [2,3] and the Large Hadron Collider [4], on which traditional methods of particle acceleration are used, are very close to the limit of mankind's capability, even if the joint efforts would be applied [5]. The first recognition of the "end of the road" for traditional acceleration schemes was declared in work [6], where the ideas of new acceleration methods were proclaimed. In particular, it was a proposition made by Ya.B. Fainberg to use plasma waveguides as accelerating structures. Later, this idea was modified by J.M. Dawson et al. [7, 8] into a scheme of wakefield acceleration. According to it, the role of a highgradient accelerating field is played by a wake field that is excited in plasma by a short and powerful laser pulse or by a short electron bunch with a large charge.

Another probable candidate for the future highgradient particle accelerators, which is capable to overcome the acceleration threshold for ordinary accelerators $(100 \mathrm{MeV} / \mathrm{m})$, is an accelerating structure loaded with a dielectric [9], in which the wake field is excited by an intensive electron bunch or a sequence of bunches. As was shown in the theoretical [10] and recent experimental [11] works, the maximum accelerating field in the dielectric structures,

ISSN 2071-0194. Ukr. J. Phys. 2016. Vol. 61, No. 8 
which is restricted by the electric breakdown owing to the tunnel ionization, reaches $1 \mathrm{GeV} / \mathrm{m}$, which is an order of magnitude higher in comparison with the ordinary metallic accelerating structures. In addition, the dielectric structures are more acceptable owing to their simple design, the developed technology of their microfabrication, homogeneity, a possibility of HFmatching, and so forth.

In works $[12,13]$, the wakefield excitation using a sequence of bunches in a dielectric structure with a vacuum channel for the bunch passage was studied. In this work, the concept of a multibunch plasmadielectric wakefield accelerator with a vacuum channel filled by plasma, which results in the excitation of an additional plasma wakefield, is considered theoretically (by applying a numerical simulation) and experimentally. The plasma field makes it possible to enhance the efficiency of the longitudinal wakefield excitation and ensure the transverse stability of exciting and accelerated bunches. In Section 2, the superposition of coherent wakefields excited by bunches in a sequence is considered. It is required to realize the multibunch excitation regime, in which the total excited field is proportional to the number of exciting bunches. In Section 3, we demonstrate a capability for certain bunches to be accelerated by the wakefields generated by previous bunches in the same sequence making no use of an additional acceleratorinjector. This effect can be obtained by detuning the bunch repetition frequency with respect to the frequency of the excited wakefield. In Section 4, the advantages of the hybrid plasma-dielectric structure are analyzed. Unlike the results obtained in works $[12,13]$, the excited wake wave affects the total accelerating and focusing fields, which makes it possible to accelerate the bunches by the field of a dielectric wake wave (below, the "dielectric wakefield") and to focus the exciting and accelerated bunches by the field of the plasma wake wave (below, the "plasma wakefield"). The wave-guide and resonator cases are considered. In particular, for a certain waveguide segment, the number of the bunches, whose coherently summed-up fields increase the total field, is confined owing to the wakefield outlet at a group velocity from the waveguide end. In the case of resonator, the excited wakefields of separate bunches are reflected from the metallic ends of the resonator and are accumulated in the latter. The necessary requirement consists in that the characteristic frequencies of the res-

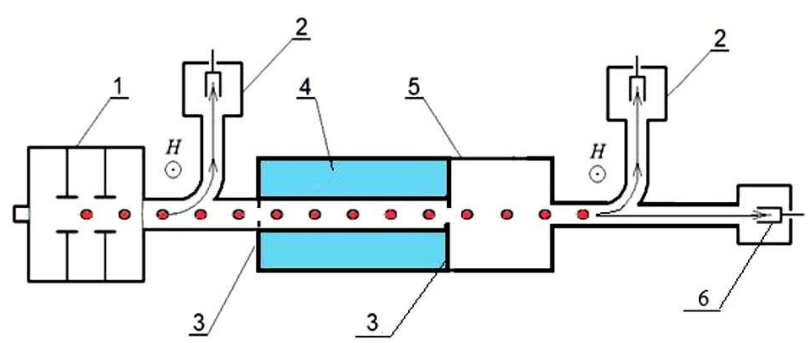

Fig. 1. Schematic diagram of the experimental installation: accelerator "Almaz-2M" (1), magnetic analyzers (2), input and output diaphragms (3), teflon tube (4), metallic wave guide $(5)$, and Faraday cup $(6)$

onator should coincide with (or be multiple of) the wakefield (Cherenkov) and bunch repetition frequencies. In the same section, an experimental demonstration of the focusing of exciting bunches, when plasma fills the transit channel, is shown.

\section{Wakefield Excitation}

\subsection{Experimental equipment}

As a result of the works on the restoration of a klystron amplifier and the modernization of an electron gun and a master oscillator on the linear resonant electron accelerator "Almaz-2M", we obtained a beam of relativistic electrons, the energy of which could be varied from 2.5 to $4.8 \mathrm{MeV}$, and the current in a pulse from 0.5 to $1 \mathrm{~A}$ at a pulse duration of $2 \mu \mathrm{s}$. Every pulse was a sequence of $N=6000$ electron bunches. The duration of each of them equaled $60 \mathrm{ps}$, and an interval between them was 300 ps (if the bunch repetition frequency was $2805 \mathrm{MHz}$ ). The bunch charge varied from 0.16 to $0.32 \mathrm{nC}$. The bunch repetition frequency could be changed within the limits from 2803 to $2807 \mathrm{MHz}$. The energy spectrum width varied from $7 \%$ to $9 \%$. The accelerator "Almaz$2 \mathrm{M}$ " was equipped with a chamber, in which a dielectric structure could be arranged. As a result, we obtained an experimental installation for researching the wakefield excitation by a sequence of relativistic electron bunches in dielectric structures with a round or rectangular transverse cross-section and the acceleration of electrons by excited wakefields. The experimental setup is illustrated in Fig. 1.

As a dielectric structure, we took a copper cylindrical waveguide 5 with an internal diameter of $85 \mathrm{~mm}$ and filled with a dielectric tube 4 . The latter was fabricated of teflon $\left(\varepsilon=2.04\right.$ and $\left.\tan \delta=4 \times 10^{-4}\right)$, and 

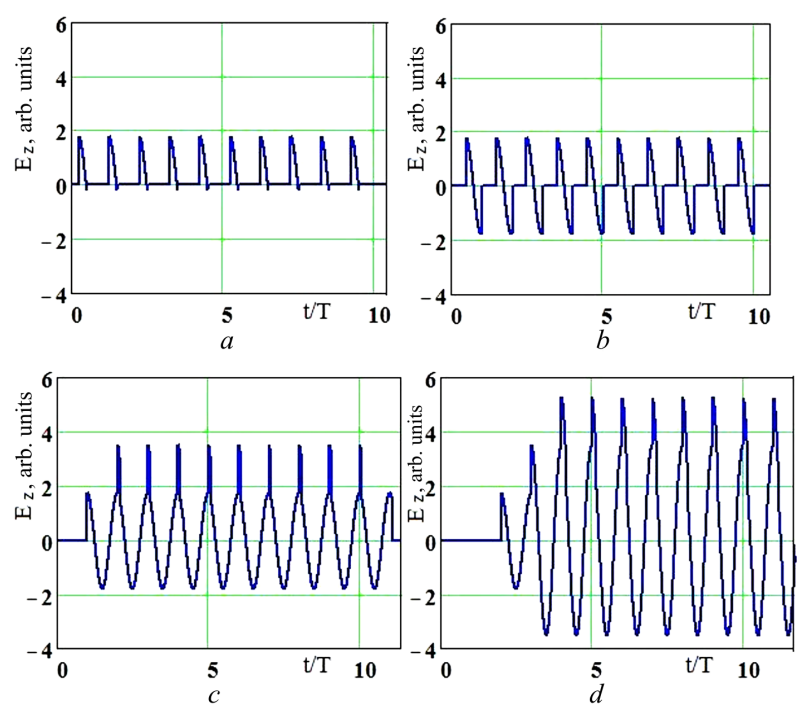

Fig. 2. Time dependences of the Cherenkov wakefield at the output end of the dielectric waveguide with the lengths $L$ : (a) $\lambda / 4,(b) \lambda / 2,(c) \lambda$, and $(d) 2 \lambda$. The number of bunches $N=10$

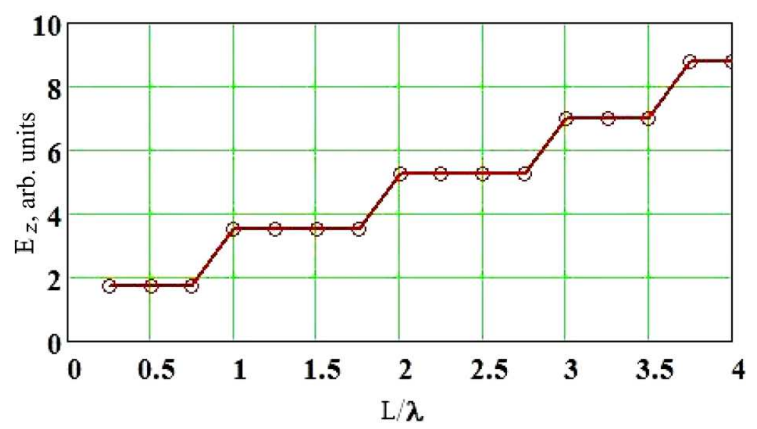

Fig. 3. Dependence of the Cherenkov wakefield at the output of the waveguide on the waveguide length

its external diameter was equal to the internal diameter of the copper waveguide. The internal part of the tube was a channel $2.1 \mathrm{~cm}$ in diameter for the passage of electron bunches. The length of the waveguide section filled with the dielectric was equal to $31 \mathrm{~cm}$, which corresponded to three wavelengths of the principal mode. The energy spectra of electrons in the bunches were measured with the help of magnetic analyzers 2 located at the outputs of the accelerator and the dielectric structure. The wakefields were registered with the use of a high-frequency probe.

\section{2. "Multibunch" excitation}

The "multibunch" approach consists in that the intense wakefield excited by a bunch with a large charge can be produced by a long periodic sequence of bunches, every of them with a small charge, but with an equivalent total charge. In order to clarify whether it would be possible to coherently sum-up the fields excited by separate bunches, it is necessary to change the number of bunches in the sequence. Because of the difficulties in obtaining the sequences with various numbers of bunches, we used slow-wave guides of various lengths in our researches. A possibility of this replacement follows from the fact that, since the excited wave is characterized by the group velocity $v_{g}$, its outlet from a waveguide with a finite length confines the number of bunches in the sequence that give a contribution to the growth of the total wakefield at the waveguide output. The maximum number $N$ of bunches, whose Cherenkov wakefields, being coherently summed up, increase the amplitude of the total Cherenkov field, depends on the waveguide length $L: N=L / \lambda\left(\left(v_{0} / v_{g}\right)-1\right)$, where the length of the excited wave $\lambda$ is equal to the distances between the bunches, $v_{0}$ is the bunch velocity, and $v_{g}$ is the group velocity of the excited wave. The time dependence of the excited field at the output of the dielectric waveguide is shown in Fig. 2 for $v_{g}=v_{0} / 2$ and various waveguide lengths $L=\lambda / 4$, $\lambda / 2, \lambda$, and $2 \lambda$. One can see that the field amplitude does not change until $L<\lambda$. As the waveguide length increases, the wakefield grows in a jump-like manner, with the period $\lambda$ between the jumps (see Fig. 3).

In order to consider the transient radiation emission, which inevitably arises at electrodynamic jumps in real structures, and reflections of excited fields, which occur owing to the imperfect matching between the waveguides, a numerical simulation of a model dielectric structure close to the experimental one was carried out. In Fig. 4, the obtained dependence of the total excited field on the length of the dielectric part in the structure is shown. The maxima and minima in this dependence are associated with two factors. First, if the dielectric length is multiple of the half wavelength in the resonance mode, the reflection of the wave excited by a bunch is minimum at the dielectric-vacuum interface. Second, the partially reflected wave coherently interacts with the direct wave, so that the total field in the dielectric segment increases, which gives rise to the growth of the excited field at the next wave incidence on the interface between the media. This situation with the 


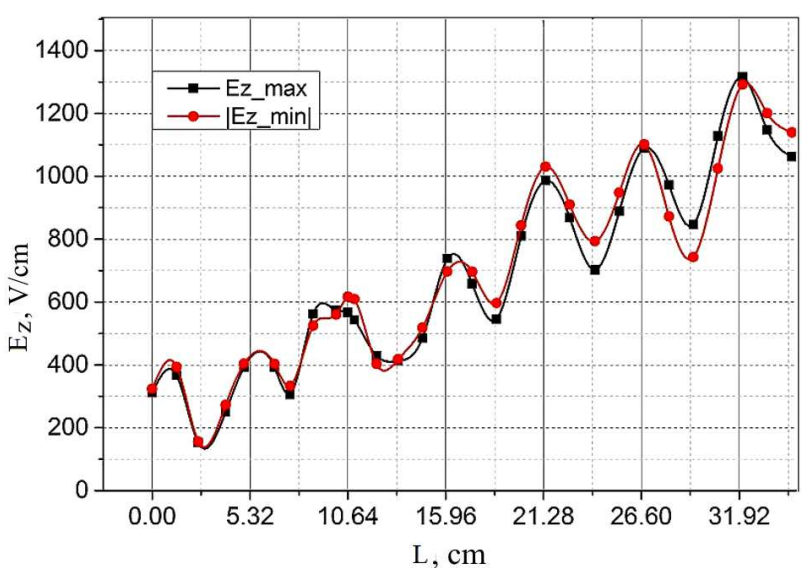

Fig. 4. Maxima and minima of the longitudinal electric field at the waveguide output at various lengths of the dielectric part

field growth is similar to the energy accumulation in a resonator.

A linear, on average, growth of the total wakefield excited in the dielectric waveguide by a long bunch sequence with increase of the waveguide length was experimentally obtained (the waveguide length was changed from 0 to $35 \mathrm{~cm}$ with a step of $2.5 \mathrm{~mm}$ ). This result testifies to the coherent summation of wakefields that overlap as the waveguide length increases (Figs. 5 and 6). The measured magnitudes of wakefields excited by one to four bunches agree with the results of theoretical calculations and numerical simulations.

When a long sequence of bunches was used in the case of an unmatched waveguide, then, owing to the reflection, the additional fields with resonance peaks near the waveguide lengths that are multiple of the excited half wavelength were observed against the background of a linearly growing wakefield (Fig. 5). The improvement of the waveguide matching (adiabatic adapters and adsorbers), which reduce the wave reflection, brought about a reduction of the wakefield resonance peaks (Fig. 6) and gave rise to a satisfactory coincidence with the results of a numerical simulation (Fig. 4).

In case of the dielectric structure with a rectangular cross-section, there emerges a possibility to equalize the reflection effect for different lengths of interaction of a bunch with the dielectric part. This possibility is realized by deviating the bunches, with the help of a magnetic field, to the lateral walls of

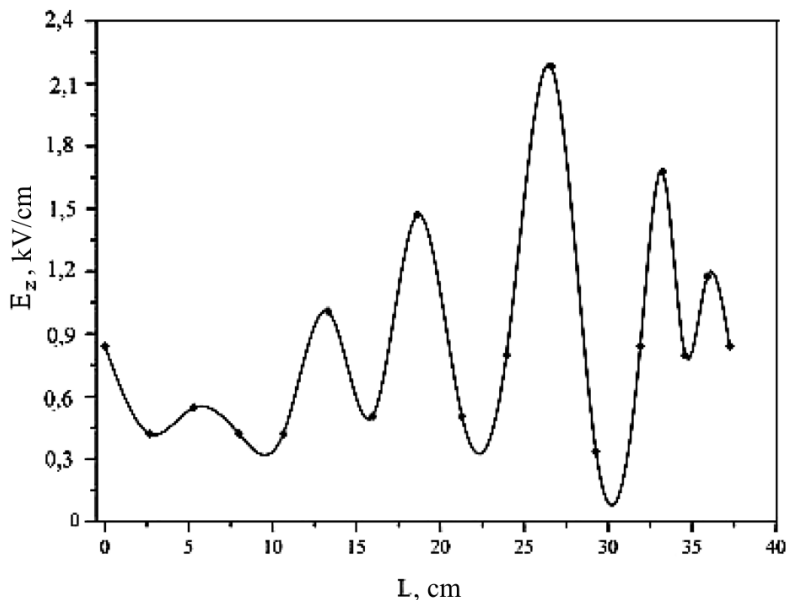

Fig. 5. Dependence of the amplitude of the $E_{z}$-component of excited wakefield on the length of an unmatched dielectric waveguide

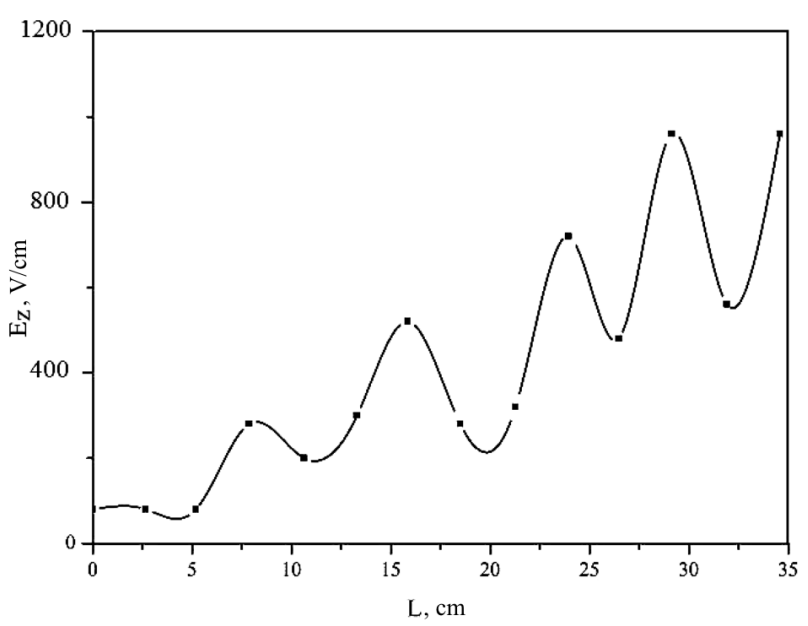

Fig. 6. Dependence of the amplitude of the $E_{z}$-component of the excited wakefield on the length of a matched dielectric waveguide

the waveguide, where the dielectric plates are absent (see Fig. 7). The shift of magnets changes the interaction length, but leaves the reflection conditions invariable.

In this experiment, we obtained a linear dependence of the wakefield amplitude on the length of interaction of a bunch with the dielectric (Fig. 8). Together with the corresponding theoretical calculations, this result confirms the coherent summation of bunch wakefields. 


\section{3. "Injection" Problem of Bunch Acceleration}

The "injection" problem was solved by introducing a detuning between the bunch repetition frequency and the principal mode frequency of the excited wakefield. This trick allowed the exciting and accelerated bunches to be obtained from the same sequence. For the case of non-zero detuning, the theoretical and experimental researches of the wakefield excitation in a dielectric waveguide/resonator by a periodic sequence of driving electron bunches and the acceleration of other bunches from the same sequence (witness bunches) by this field were carried out. This possibility arises owing to a gradual shift of the subsequent bunches with respect to the phase of the wakefield excited in front of them. In our experiments, the wakefield frequency was fixed, being determined by the Cherenkov resonance, i.e. a coincidence between the bunch velocity and the phase velocity of the excited wave in the di-

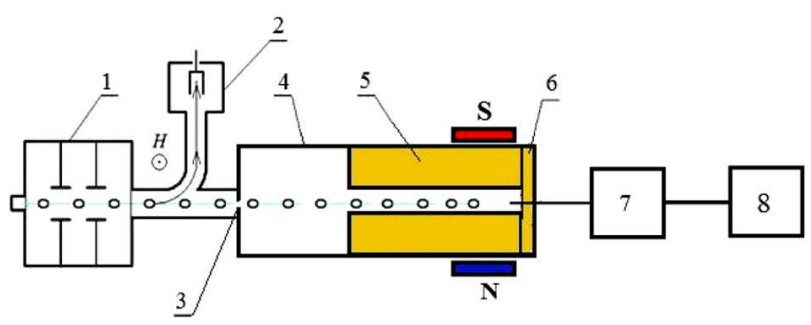

Fig. 7. Accelerator "Almaz-2M" (1), magnetic analyzer (2), diaphragm (3), wave guide (4), two dielectric plates (5), dielectric plug (6), wavemeter VMT-10 (7), and oscillograph (8)

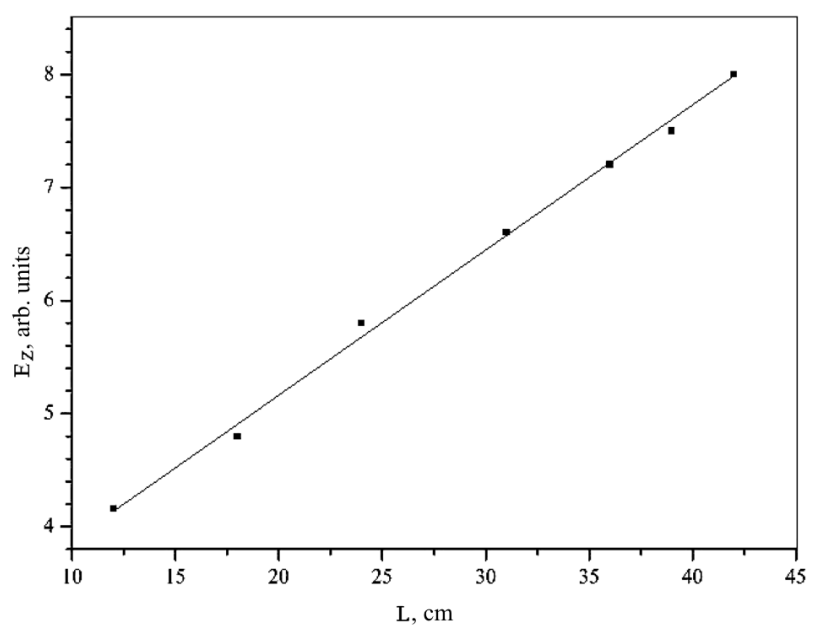

Fig. 8. Dependence of the wakefield amplitude on the length of interaction between the bunches and the dielectric waveguide electric wave guide. The bunch repetition frequency was changed by varying the frequency of the master oscillator "Rubin" of a klystron amplifier. In the framework of the concept "excitation-acceleration" for the same sequence of bunches, the necessity in an additional accelerator-injector for obtaining the witness bunches disappears, which simplifies the experimental demonstration of bunch acceleration in excited wakefields.

In the case of resonance where the bunch repetition frequency $f_{\text {rep }}$ and the principal mode frequency of excited wakefield $f_{0}$ coincide $\left(f_{\text {rep }}=f_{0}\right)$, all bunches turn out in the decelerating phase and spend their energy for the wakefield excitation. If the detuning is non-zero, $\Delta f=f_{\text {rep }}-f_{0} \neq 0$, the bunches in the first part of the sequence, which are in the decelerating phases of the excited field, lose energy to increase the total wakefield. At the same time, the bunches in the next part of the sequence, which become shifted into the region of accelerating phases of the wakefield created by the bunches in the first part of the sequence, obtain an additional energy.

For point-like and monoenergetic bunches, the number $N^{*}$ of bunches in the first part of the sequence, which excites the wakefield, is determined from the condition that the phase shift of the $N^{*}$-th bunch should be equal to $\pi$, i.e. $N^{*}=f_{\text {rep }} / 2 \Delta f$. The bunches in the next part of the sequence characterized by the same duration are accelerated. As a result, the initially monoenergetic spectrum of the sequence becomes split into two components: for the decelerated (energy loss on excitation) and accelerated (an energy gain in the wakefield) beams.

In order to accelerate the bunches in our experiments more substantially, the wakefield amplitude had to be additionally increased. In the waveguide case, the total wakefield is determined by the sum of the fields generated by only a finite number of bunches. This number depends on the waveguide length (see section 2.2). Under the experimental conditions, only a few bunches are involved. Therefore, in order to increase the wakefield amplitude, a resonator mode was applied. It consists in that the reflection of excited fields from resonator's ends allows the wakefields of a larger number of bunches to be summed up. The restriction on the number of bunches that give a contribution to the total field is determined only by the resonator $Q$-factor: $N=Q / \pi$.

ISSN 2071-0194. Ukr. J. Phys. 2016. Vol. 61, No. 8 
In Fig. 9, the experimental energy spectra of electrons in the sequence of bunches that passed through the resonator without the dielectric - so that there is no Cherenkov interaction between the bunches and the resonator (these spectra are marked by squares; their form is close to the initial spectrum at the resonator input) - and through the resonator with a dielectric tube (these spectra are marked by circles; they correspond to the electron interaction with the excited wakefield) are exhibited for two cases: at the resonance (panel $a, \Delta f=0$ ) and without it (panel $b$, $\Delta f=2.5 \mathrm{MHz})$.

From Fig. 9, it follows that, with the dielectric, the whole energy spectrum shifts by $400 \mathrm{keV}$ toward lower energies at the resonance, $\Delta f=0$, which is associated with the energy losses by all bunches for the wakefield excitation. In the case of a detuning between the bunch repetition frequency and the wakefield frequency, $\Delta f=f_{\text {rep }}-f_{0}=2.5 \mathrm{MHz}$, some of the bunches in the sequence, undergoing the phase shift, find themselves in the accelerating phase of the wakefield excited by previous bunches in the same sequence and absorb the wakefield energy. In this case, the energy spectra reveal the presence of the electrons that lost energy $(-150 \mathrm{keV})$ and the electrons accelerated to an energy higher than the initial one $(+150 \mathrm{keV})$.

By varying the detuning value, it is possible to regulate the number of bunches in the part of the sequence that excites the wakefield, as well as the number of bunches that turn out in the accelerating wakefield phase and obtain an additional energy. If the detuning grows, there arise conditions for the emergence of wakefield beats with several parts in the sequence of decelerated and accelerated bunches.

\section{Plasma-Dielectric Structure}

The presence of plasma in the transit channel for bunches makes it possible to compensate the bulk charge of bunches and to prevent the electron bunches from reaching the dielectric. As a result, the conditions for the beam to pass through the channel become better, which increases the amplitude of the excited wakefield at the output. In addition, plasma changes the dispersion characteristics of the waveguide/resonator and the topography of the wakefield excited by the bunch sequence at the Cherenkov resonance in the channel.

ISSN 2071-0194. Ukr. J. Phys. 2016. Vol. 61, No. 8
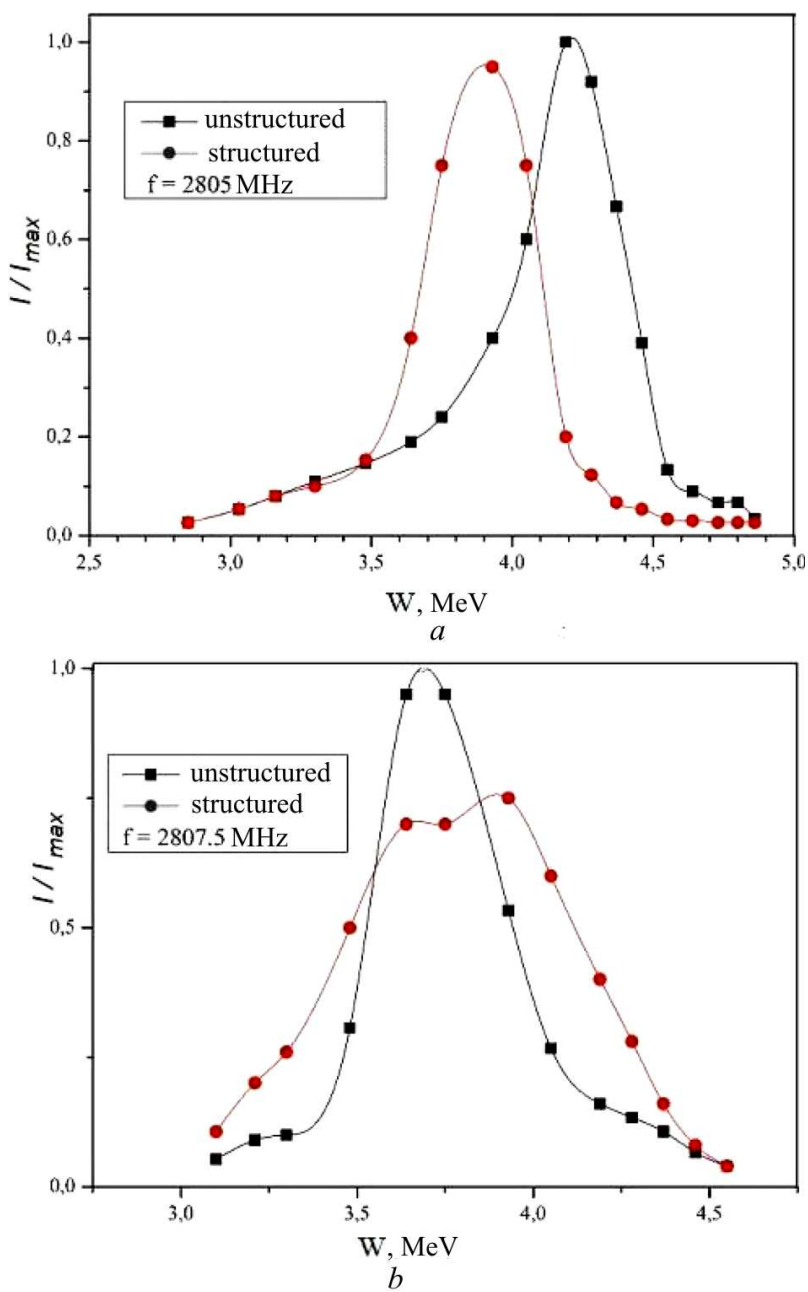

Fig. 9. Energy spectra of electron bunches passing through the resonator without an dielectric (squares) and through the resonator with a dielectric tube (circles): ( $a) \Delta f=0$ and $(b)$ $\Delta f=2.5 \mathrm{MHz} . I$ is the current from the Faraday cup of a magnetic analyzer

Note that the wakefield consists of a plasmamodified dielectric field and a pure plasma one. Those fields are excited at different frequencies, but for the case where the plasma frequency $f_{p}$ and the frequency of modified dielectric field $f_{0}$ coincide $\left(f_{p}=f_{0}\right)$. In the scheme with a single driving bunch and a single witness bunch, the application of different frequencies allows the latter to be arranged in the phase region, where it will be accelerated by a high longitudinal dielectric field (the radial field is insignificant in the case of relativistic bunches) and focused by a high plasma radial field (Fig. 10). 

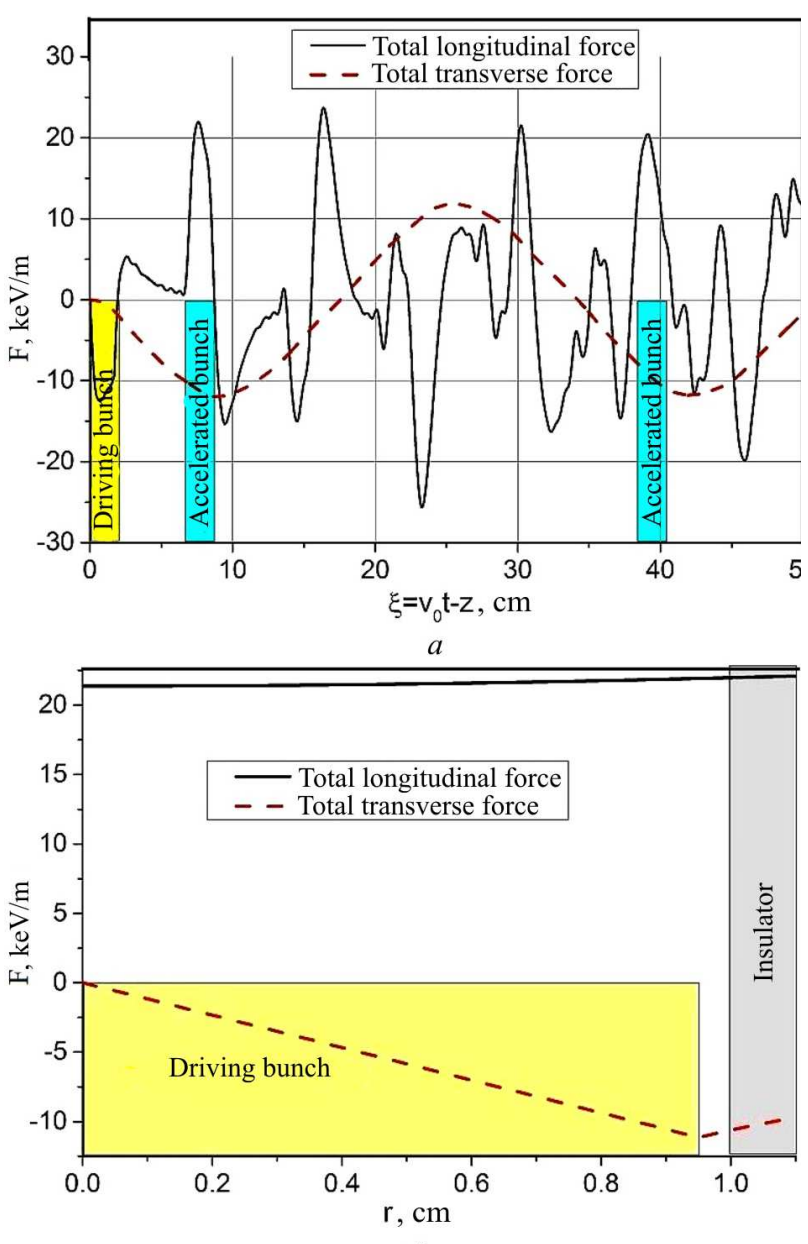

$b$

Fig. 10. Axial profile of the longitudinal (solid curve) and transverse (dashes curve) forces acting on a test particle at a distance of $0.95 \mathrm{~cm}$ from the waveguide axis $(a)$; transverse profile of the longitudinal (solid curve) and transverse (dashes curve) forces acting on a test particle at a distance of $7.56 \mathrm{~cm}$ from the head of the leading bunch $(b)$

Our theoretical researches concerning the electrodynamics of a dielectric waveguide with an axial transit channel filled with plasma (a hybrid plasmadielectric waveguide) demonstrated that the presence of plasma in the transit channel gives rise to the change in the principal mode topography for the dielectric wakefield, so that the field becomes three-dimensional in the channel with $r=0 \div 1 \mathrm{~cm}$ (Fig. 11). The corresponding increase of the coefficient of coupling of a bunch with the wave provides a growth of the longitudinal field amplitude in the channel with the plasma density.

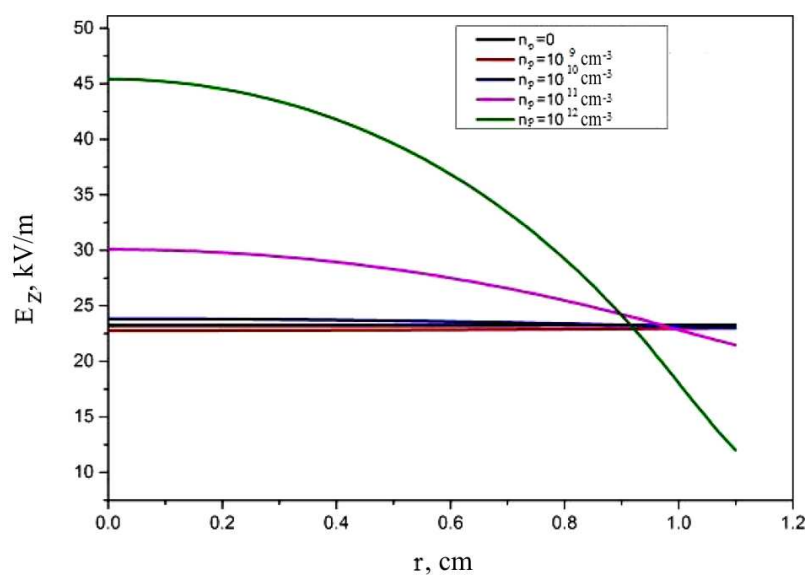

Fig. 11. Topography of the wakefield excited by a single bunch in the transit channel of a dielectric waveguide with a round cross-section for various plasma densities

For a sequence of bunches, the situation becomes complicated, because plasma in the channel violates the resonance condition of the coincidence between the bunch repetition frequency $f_{\text {rep }}$ and the frequency of the excited Cherenkov dielectric field $f_{0}$. As a result, the presence of plasma gives rise to the total field beats, as the number of bunches increases. This circumstance confines the linear growth of the field with the bunch number, and the maximum field for a long sequence of bunches considerably decreases in comparison with the case where the channel is free of plasma. The situation becomes more complicated in the resonator case, because the condition for the additional resonance with the characteristic resonator frequencies $f_{n}$ has to be satisfied, i.e. $f_{\text {rep }}=f_{0}=f_{n}$.

In order to experimentally confirm our conclusions on the role of the presence of plasma in the transit channel (the enhancement or suppression of the wakefield excitation), we carried out experiments with a resonator, in which plasma suppresses the excitation owing to the emergence of the resonance detuning, $f_{\text {rep }} \neq f_{0} \neq f_{n}$, and with a waveguide, in which, according to the theory (Fig. 11), the presence of plasma should induce a growth of the excited field in comparison with the plasma-free case. In the case of waveguide configuration, it is necessary to exclude the influence of the bunch repetition frequency $f_{\text {rep }}$, which, in the presence of plasma, gives rise to a detuning with the frequency of the Cherenkov dielectric wakefield $f_{0}\left(f_{\text {rep }} \neq f_{0}\right)$. For this purpose, it is nec-

ISSN 2071-0194. Ukr. J. Phys. 2016. Vol. 61, No. 8 
essary to change to the case with a single bunch. As was shown above, a single bunch can be obtained under our experimental conditions $\left(v_{g}=v_{0} / 2\right)$, if the waveguide length $L$ is chosen to equal the dielectric wave length $\lambda(L=\lambda)$. In this case, the bunches excite the wakefield independently. Every bunch takes away the field excited by itself from the waveguide, so that the next bunch enters the waveguide free of the fields created by previous bunches. Therefore, the envelope of the fields of all $6 \times 10^{3}$ bunches has an amplitude (this amplitude can be measured in experiment) equal to the amplitude of the field excited by a single bunch, and a comparison with the theory (see Fig. 11) becomes correct.

\subsection{Experimental installation}

The diagram of the experimental installation is shown in Fig. 12. Relativistic electron bunches were injected into a dielectric waveguide through a $30-\mu \mathrm{m}$ titanic foil. The waveguide had a round cross-section filled with an dielectric with a transit channel $21 \mathrm{~mm}$ in diameter for the bunch passage.

In order to obtain a waveguide, we had to exclude the reflection of the excited wave. For this purpose, the dielectric inset ended as a dielectric cone, and a ferrite absorber was mounted on a dielectric vacuum plug. For the single-bunch regime to be obtained, the dielectric inset length $L$ was selected to equal the excited dielectric wavelength $\lambda$.

Plasma was created in the transit channel of the dielectric waveguide by the beam itself at its passage through a neutral gas with controllable pressure, which filled the transit channel. Plasma emerged owing to the development of the beam-plasma discharge in the excited wakefield at a pressure of about 1 Torr and to the impact ionization by beam electrons at high pressures.

To study the focusing of relativistic electron bunches, dual Faraday cup (9) was used. The bunch focusing was registered by the current increase at the second cup and a simultaneous current reduction at the first cup.

\subsection{Influence of the presence of plasma in the transit channel on the efficiency of wakefield excitation}

Oscillograms of the microwave wakefield signal were obtained with the help of a microwave probe mounted

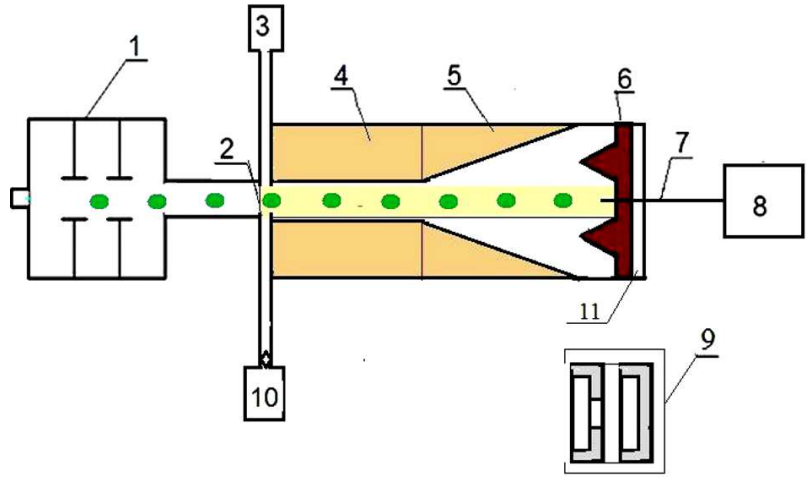

Fig. 12. Schematic diagram of the experimental installation: (1) accelerator "Almaz-2M" (2) titanic foil, (3) vacuum gauge, (4) dielectric waveguide, (5) matching dielectric cone, (6) ferrite absorber, (7) microwave probe, (8) oscillograph GDS-840C, (9) dual Faraday cup, (10) vacuum pump, and (11) dielectric vacuum plug

at the output of the dielectric waveguide with a dielectric inset of the length $L=\lambda$ (Fig. 13). The pressure of a neutral gas in the transit channel varied from 0.02 to 1 Torr. The oscillograms demonstrate that the amplitude of the excited wakefield at a pressure of 0.5 Torr, when plasma is formed (panel $b$ ), exceeds the amplitude of the field in the dielectric waveguide at pressures, when plasma is not formed (panels $a$ and $c$ ).

The dependence of the excited longitudinal wakefield amplitude at the transit channel axis on the pressure in the whole examined pressure interval in the waveguide configuration and in the single-bunch regime is depicted in Fig. 14 (curve 1). One can see that the wakefield wave in the channel at pressures, at which the beam-plasma discharge develops and plasma is formed, becomes three-dimensional (in agreement with the theory (Fig. 11)). As a result, the coefficient of coupling of a bunch with the wave increases, and the excited wakefield amplitude grows in comparison with the no-gas case (Fig. 14, the lower horizontal line).

The configuration with a dielectric resonator was obtained by removing the matching elements and mounting a metallic plug at the output. Under the double resonance conditions $f_{\text {rep }}=f_{0}=f_{n}$ (the coincidences of the Cherenkov frequency $f_{0}$ with the bunch repetition frequency $f_{\text {rep }}$ and, simultaneously, with the characteristic resonator frequency $f_{n}$ ), the wakefield amplitude was found to grow substan- 


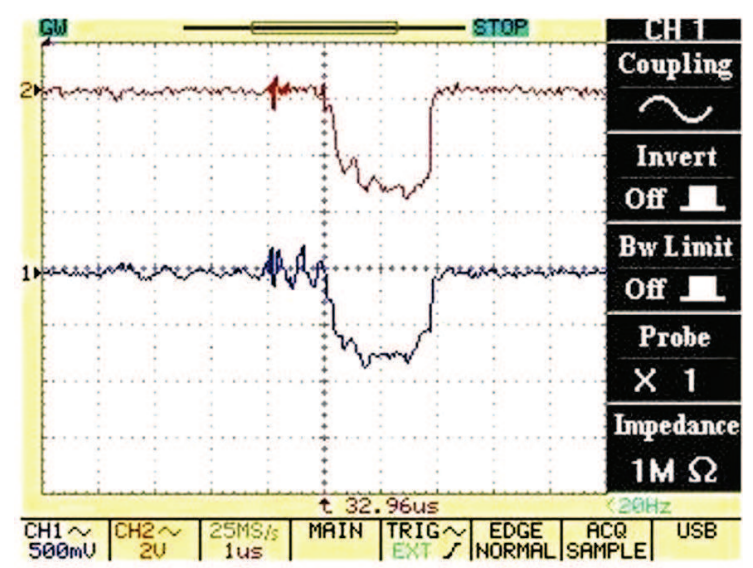

$a$

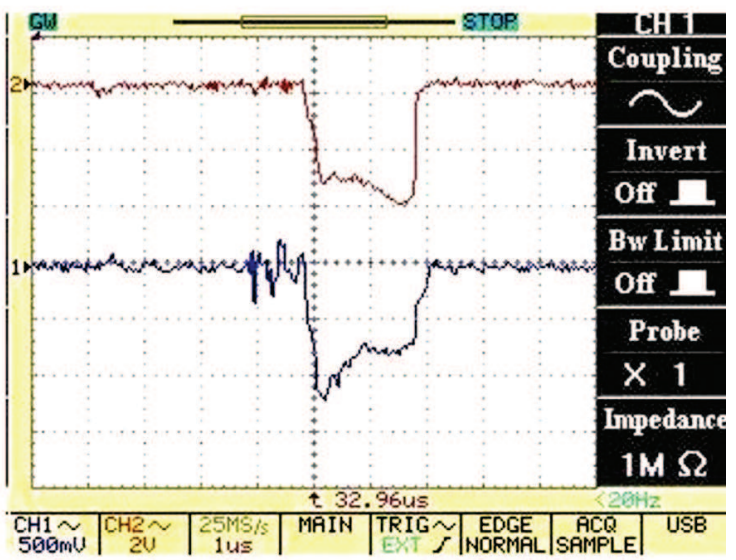

$b$

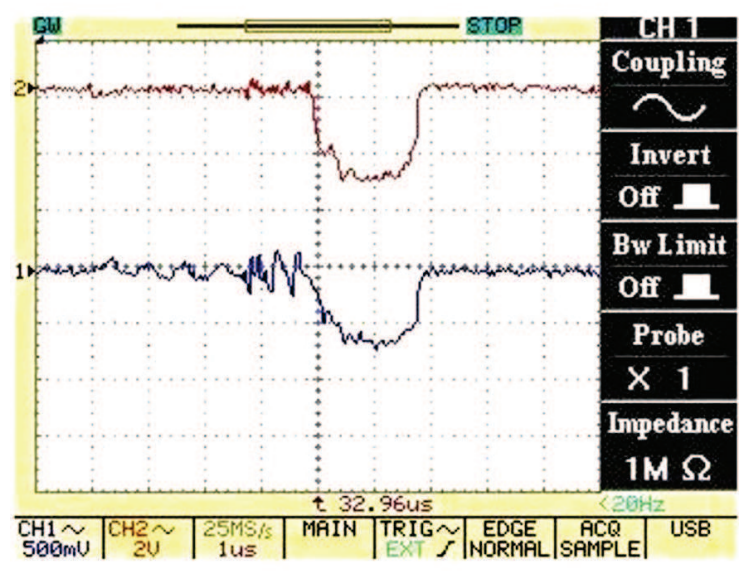

$c$

Fig. 13. Oscillograms of the envelope of the wakefield microwave signal (lower oscillograms) at $P=10^{-3}(a), 0.5(b)$, and 140 Torr $(c)$. Upper oscillograms correspond to the beam current

698

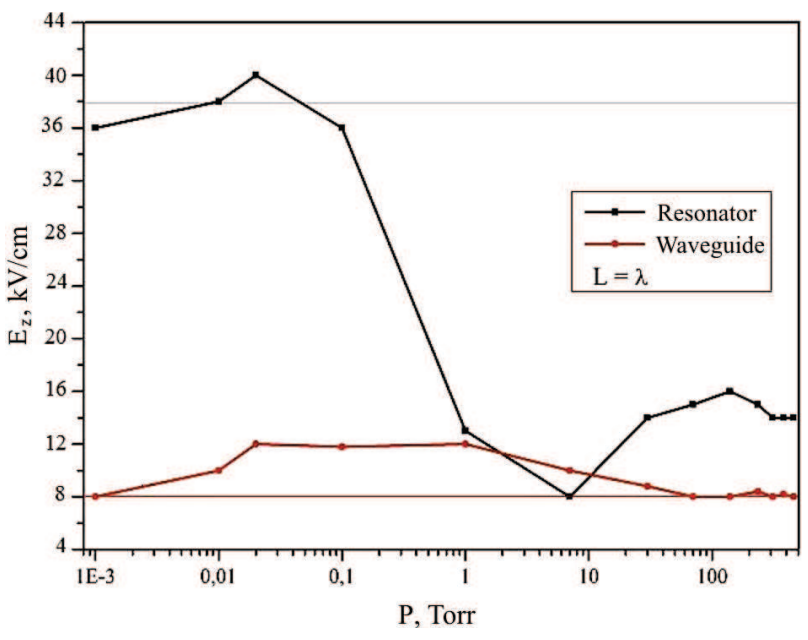

Fig. 14. Dependences of the $E_{z}$-component of the excited field on the pressure of a neutral gas in various dielectric structures

tially. This phenomenon results from the fact that the number of bunches that give a contribution to the total wakefield is connected with the $Q$-factor and, for real $Q$-values, amounts to several hundreds. At the same time, in the waveguide configuration, the corresponding number of bunches, which is determined by the waveguide length and the group velocity, does not exceed several tens.

However, in contrast to the waveguide configuration with a single bunch, in the case of resonator, many bunches are involved into the wakefield excitation, i.e. the bunch repetition frequency $f_{\text {rep }}$ begins to play a certain role, and there emerge characteristic resonator frequencies $f_{n}$. The presence of plasma at pressures, at which the beam-plasma discharge develops, results in the detuning of both resonances and a reduction of the wakefield amplitude (Fig. 14, curve 2), unlike the no-gas, i.e. no-plasma, case (Fig. 14, the upper horizontal line).

\subsection{The focusing of driving bunches}

In the waveguide geometry (the matched output) and the single-bunch regime $(L=\lambda)$, both mentioned resonances are absent, so that all bunches are under identical conditions of exciting driving bunches. In Fig. 15, the calculated wakefield profiles of the dielectric and plasma waves excited by a single bunch are shown for two plasma densities [14]. One can see that the bunch with a finite length and a finite radius is located in its own wakefield, the longitudinal 

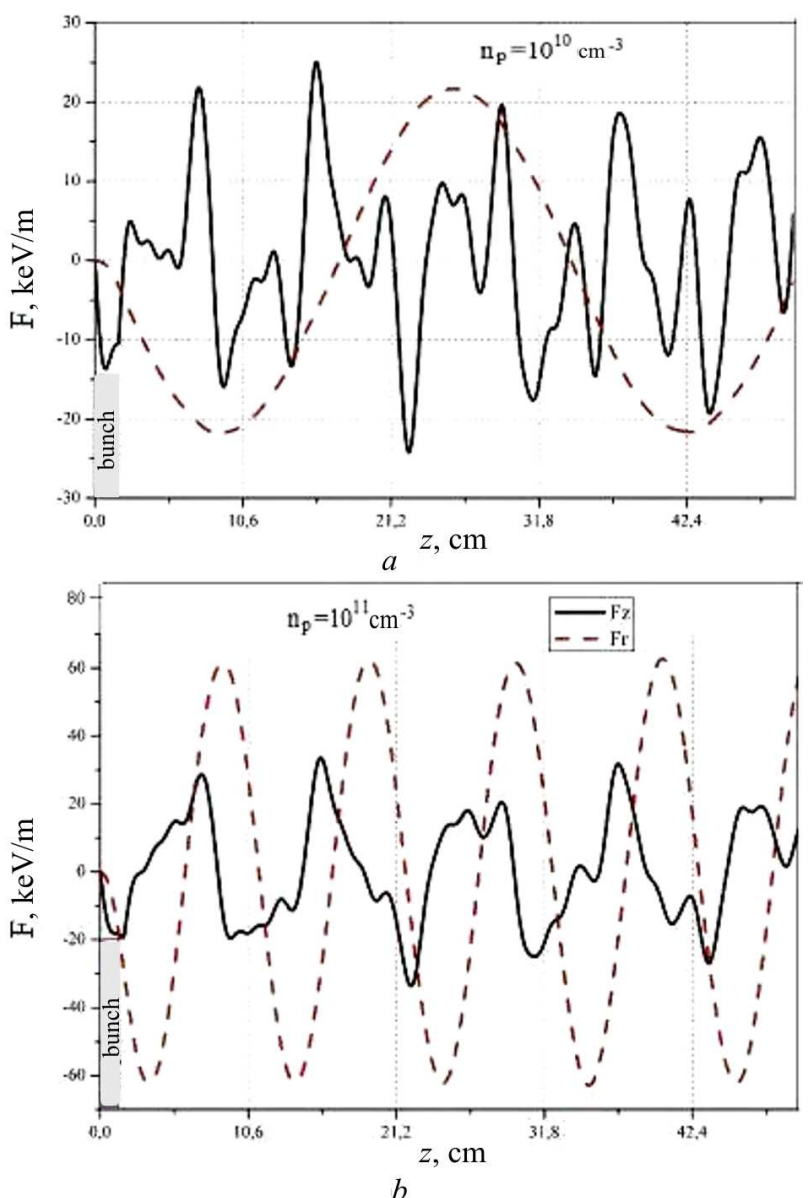

Fig. 15. Summed-up longitudinal components of the plasma and dielectric waves (solid curve) and the transverse component of a plasma wave (dashed curve) at various plasma densities $n_{p}=10^{10}(a)$ and $10^{11} \mathrm{~cm}^{-3}(b)$

dielectric (decelerating) and radial plasma-generated (focusing) ones. There is no radial defocusing field of the dielectric wave, and its longitudinal field is almost uniform. As a result, the driving bunch is focused by its own wakefield excited in plasma, in addition to the focusing associated with the compensation of its radial electric field in plasma.

In Fig. 16, experimental oscillograms of the beam current obtained with the help of a dual Faraday cup in vacuum with $P=10^{-3}$ Torr (panel $a$ ) and at the pressure $P=0.5$ Torr of a neutral gas in the transit channel of the dielectric waveguide (panel b) are shown. The current growth at the second cup together with the simultaneous current reduction at the first cup testifies to the stronger focusISSN 2071-0194. Ukr. J. Phys. 2016. Vol. 61, No. 8
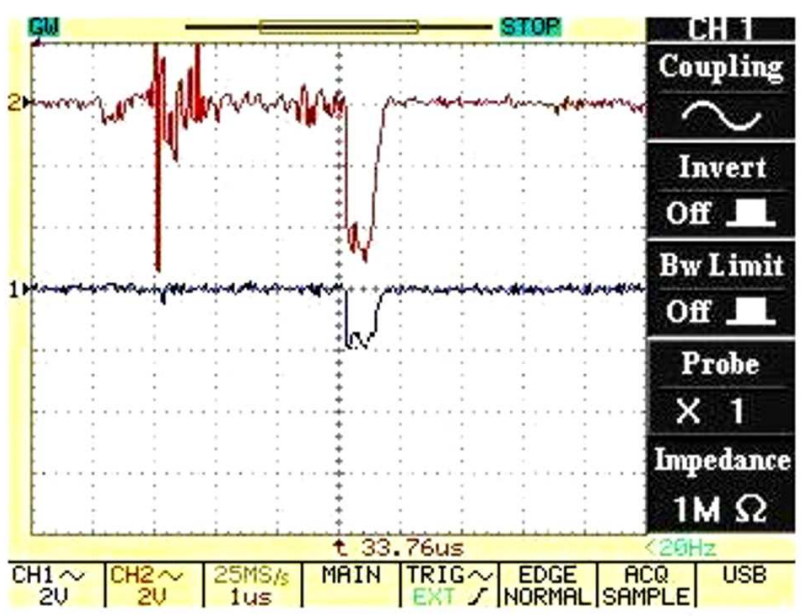

$a$

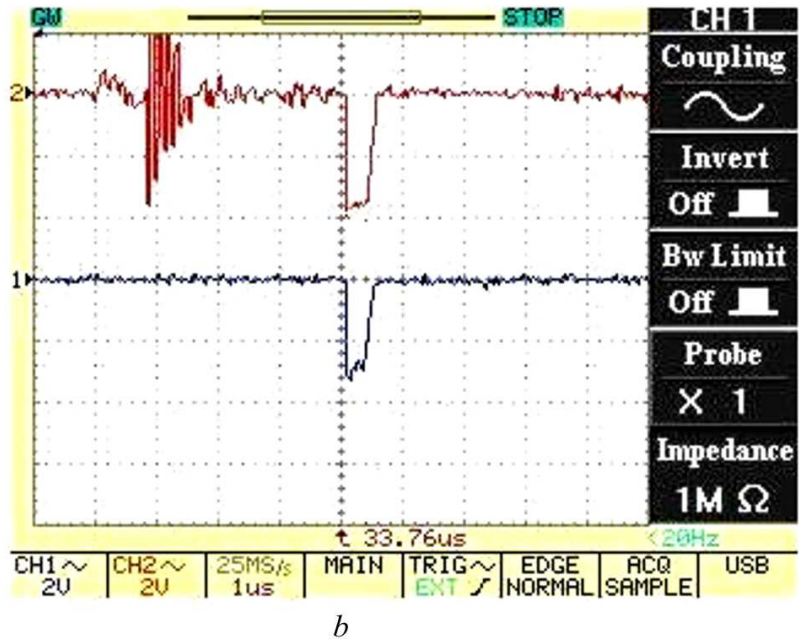

Fig. 16. Oscillograms of the beam current registered with the help of a dual Faraday cup (the upper curve corresponds to the first cup, and the lower one to the second cup) and the oscillograph GDS-840C at various pressures $P=10^{-3}(a)$ and 0.5 Torr $(b)$

ing of electron bunches in the latter case corresponding to a higher plasma density at the gas pressure $P=0.5$ Torr.

\section{Conclusions}

Using theoretical calculations and numerical simulations, it is shown that when a wakefield (the Cherenkov radiation) is excited by a sequence of relativistic electron bunches in dielectric structures, and the bunch repetition frequency coincides with the wakefield frequency, the total wakefield, owing 
to the coherent summation of the wakefields generated by separate bunches, can reach the amplitudes of the wakefield excited by a single bunch with an equivalent total charge. This circumstance makes it possible to apply a regular sequence of bunches produced at ordinary accelerators for the excitation of a high-gradient accelerating electric field, instead of intensive single bunches produced at modern colliders.

In the dielectric-waveguide configuration, the total wakefield is a contribution only of the bunches in the long sequence, the number of which is determined by the waveguide length $L$ and the group velocity $v_{g}$ of the excited wakefield: $N=L / \lambda\left(v_{0} / v_{g}-1\right)$, where $\lambda$ is the wavelength that is equal to the distance between the bunches, and $v_{0}$ is the bunch velocity. This restriction is a result of the excited wakefield outlet with the group velocity through the waveguide end.

In the dielectric-resonator configuration, all bunches in the sequence contribute to the total field owing to the reflection of the excited wakefield from the metallic input and output resonator ends. The restriction is limited only with the resonator $Q$ factor: $N=Q / \pi$. As a result, in this configuration, the total wakefield is substantially higher in comparison with that in the dielectric-waveguide configuration.

In experiments with the application of a sequence of relativistic electron bunches produced at the ordinary linear electron accelerator "Almaz-2" $(6 \times$ $10^{3}$ bunches; each with an energy of $4.5 \mathrm{MeV}$, a radius of $1 \mathrm{~cm}$, a duration of $60 \mathrm{ps}$, a charge up to $0.32 \mathrm{nC}$, and a repetition frequency of $2.805 \mathrm{GHz}$ ), the efficiency of the multibunch excitation regime for generating intensive total wakefields in the cases of waveguide and resonator configurations was demonstrated.

Taking advantage of the detuning between the bunch repetition frequency and the wakefield frequency, we obtained bunches that are accelerated by the wakefields of previous bunches in the same sequence. It enabled us to study the acceleration process making no use of a separate accelerator-injector of bunches for their acceleration by the excited wakefield.

It is shown that the presence of plasma in the transit channel of a hybrid plasma-dielectric structure results in the excitation of an additional plasma wake wave. The total wakefield in the transit channel consists of the dielectric wave field and the plasma wave field. At low plasma densities, the dielectric wave has the longitudinal field (its transverse field is negligibly low), whereas the plasma wave has the transverse field (its longitudinal field is insignificant). In the course of excitation, the bunches turn out in decelerating phases of the longitudinal dielectric wakefield and in focusing phases of the transverse plasma wakefield. The bunches intended for the acceleration can be arranged in the phases, where the amplitude of the longitudinal accelerating wakefield of the dielectric wave and the amplitude of the focusing transverse wakefield of the plasma wave are maximum. At a proper (taking the plasma response into account) choice of the parameters of a dielectric inset and the corresponding plasma density, it becomes possible to simultaneously provide an increase of the longitudinal accelerating field and the focusing of both exciting and accelerated bunches.

With the help of a dual Faraday cup, the focusing effect for the exciting bunches in a plasma-dielectric structure is experimentally demonstrated. The obtained results of theoretical and experimental researches concerning the excitation of wakefields in plasma-dielectric structures by sequences of relativistic electron bunches testify to a possibility of applying the idea of a multibunch plasma-dielectric accelerator for the future development of colliders with considerably smaller dimensions and lower cost.

The work was supported in the framework of the target complex program of the NAS of Ukraine "Promising researches in plasma physics, controlled nuclear fusion, and plasma technologies": the scientific project "Development of physical principles of plasma-dielectric wakefield accelerator. Theory and experiment" (contract No. P-1/63-2015).

1. J.R. Rees, Sci. Am. 261, 36 (1989).

2. H. Braun, R. Corsini, T D'Amico et al., CLIC-Note-364 (1998).

3. J. Brau, Y. Okada, N. Walker et al., International Linear Collider Reference Design Report (2007).

4. CERN 2008. LHC Design Report (2008) [http://abdiv.web.cern.ch/ab-div/Publications/LHC-Design Report.html].

ISSN 2071-0194. Ukr. J. Phys. 2016. Vol. 61, No. 8 
5. M. Tigner, Phys. Today 54, 36 (2001).

6. Ya.B. Fainberg, in Proc. Symp. CERN (Geneva, 1956), Vol. 1, p. 84 .

7. T. Tajima and J.M. Dawson, Phys. Rev. Lett. 43, 267 (1979).

8. P. Chen, J.M. Dawson, R. Huff, and T. Katsouleas, Phys. Rev. Lett. 54, 692 (1985).

9. W. Gai, P. Schoessow, B. Cole et al., Phys. Rev. Lett. 61, 2756 (1988).

10. P. Sprangle, B. Hafizi, and R.F. Hubbard, Phys. Rev. E 55, 5964 (1997).

11. M.C. Thompson, H. Badakov, A.M. Cook et al., Phys. Rev. Lett. 100, 214801 (2008).

12. T-B. Zhang, J.L. Hirshfield, T.C. Marshall, and B. Hafizi1, Phys. Rev. E 56, 4647 (1997).

13. I.N. Onishchenko, V.A. Kiselev, A.F. Linnik, and G.V. Sotnikov, in Proceedings of the 4th International Particle Accelerator Conference (IPAC'13), Shanghai, China, May 12-17, 2013, p. 1259.

14. R.R. Knyazev and G.V. Sotnikov, J. Kharkiv Univ. 1001, 64 (2012).

Received 07.05.2016 Translated from Ukrainian by O.I. Voitenko
Г.П. Березіна, К.В. Галайдич, Р.Р. Князєв,

А.Ф. Лінник, П.І. Марков, О.Л. Омелаєнко, І.М. Оніщенко, B.I. Приступа, Г.В. Сотніков, В.С. Ус

МУЛЬТИБАНЧЕВИЙ РЕЖИМ

ЗБУДЖЕННЯ КІЛЬВАТЕРНОГО ПОЛЯ

В ПЛАЗМОВО-ДІЕЛЕКТРИЧНІЙ СТРУКТУРІ

$\mathrm{P}$ е $з$ ю м е

Представлені теоретичні та експериментальні дослідження фізичних принципів створення діелектричного кільватерного прискорювача, заснованого на збудженні прискорюючого кільватерного поля в плазмово-діелектричній структуpi довгою послідовністю електронних згустків. Збільшення амплітуди збуджуваного кільватерного поля досягається за рахунок когерентного складання кільватерних полів окремих згустків. Прискорення згустків у сумарному кільватерному полі реалізовано поділом послідовності згустків на збуджуючу і прискорювану частини в будь-якому співвідношенні за допомогою відповідної розстройки частоти слідування згустків щодо частоти збуджуваної основної поперечної моди. Досліджено вплив плазми в прольотному каналі на величину збуджуваного кільватерного поля і фокусування збуджуючих і прискорюваних згустків. 